\title{
El empoderamiento digital en Ecuador a través de sus infocentros
}

\author{
Sara Martínez Cardama*, Mercedes Caridad Sebastián* \\ * Departamento de Biblioteconomía y Documentación/ Instituto Agustín Millares. Universidad Carlos III de Madrid \\ Correo-e: smarti1@bib.uc3m.es | ORCID iD: https://orcid.org/0000-0001-7035-5884 \\ Correo-e: mercedes@bib.uc3m.es | ORCID iD: https://orcid.org/0000-0001-7486-8353
}

Recibido: 29-09-2018; 2a versión: 18-12-2018; Aceptado: 23-12-2018.

Cómo citar este artículo/Citation: Martínez Cardama, S.; Caridad Sebastián, M. (2019). El empoderamiento digital en Ecuador a través de sus infocentros. Revista Española de Documentación Científica, 42 (3), e241. https://doi.org/10.3989/redc.2019.3.1616

Resumen: La red de infocentros de Ecuador constituye el eje vertebrador de los procesos de alfabetización informacional del país, siendo un punto esencial en el desarrollo de las Políticas TIC, por su fuerte inversión y capacidad de conectar todo el territorio. Por ello, se analiza a través de esta red la situación actual de los procesos de empoderamiento digital de la población ecuatoriana. Asimismo, este estudio evalúa su capacidad de intermediación en el proceso de empoderamiento ciudadano, especialmente orientados a colectivos específicos como mujeres, minorías indígenas y personas con diversidad funcional. Los datos para el estudio se han obtenido de la aportación de los propios responsables de los centros. Para ello, se aplicó un cuestionario a una muestra de 829 infocentros del país. La tasa de respuesta obtenida fue del 35,8\%. Los resultados muestran: su grado de desarrollo, capacidad formativa e implicación en favorecer el empoderamiento en función de distintos colectivos. Asimismo, permitió detectar problemas reales en su gestión y tendencias o líneas de acción futuras. El estudio concluye la importancia vital de estos centros para la reducción de la brecha digital en el país mediante evidencias de su utilidad formadora. Se plantean varios retos para su supervivencia: la necesaria colaboración con los agentes locales y el desarrollo de programas de empoderamiento digital para colectivos específicos. El presente estudio destaca por su carácter único y revelador y puede servir de soporte para la toma de decisiones por parte de los responsables políticos, o bien, como instrumento de reflexión y análisis para los agentes sociales implicados.

Palabras clave: Ecuador; empoderamiento; infocentros; telecentros; Políticas de Información.

\section{Digital empowerment in Ecuador through its infocenters}

Abstract: The telecenter network of Ecuador is the backbone of the country's information literacy processes, being an essential point in the development of ICT Policies due to its strong investment and ability to connect the entire territory. For this reason, the current situation of the processes of digital empowerment of the Ecuador's population is analysed through this network. Likewise, the study evaluates its capacity of intermediation in the process of citizen empowerment, especially oriented to specific groups such as women, indigenous minorities and people with functional diversity. The data for the study were obtained from the contribution of the people responsible for the centers. For this, a questionnaire was sent to a sample of 829 telecentres from the country. The response rate obtained was $35.8 \%$. The results show their degree of development, formative capacity, and involvement in favour of empowerment according to different groups. It also allowed detecting real problems in its management and future trends or lines of action. The study concludes the vital importance of these centers for the reduction of the digital divide in the country through evidences of its formative usefulness. Nevertheless, several challenges are posed for its survival, as are the necessary collaboration with local agents and the development of digital empowerment programs for specific groups. This study stands out for its unique and revealing nature, and can serve as support for decision-making by policy-makers, or as an instrument of reflection and analysis for the social agents involved.

Keywords: Ecuador; empowerment; telecenters; Information Policies.

Copyright: () 2019 CSIC. Este es un artículo de acceso abierto distribuido bajo los términos de la licencia de uso y distribución Creative Commons Reconocimiento 4.0 Internacional (CC BY 4.0). 


\section{INTRODUCCIÓN}

Uno de los indicadores clave en la evaluación de la Inclusión digital y la Alfabetización Informacional constituye el impacto social de organizaciones como infocentros y bibliotecas, por encima de la valoración cuantitativa del acceso o uso a los mismos o a la tecnología que facilitan. En Ecuador, los infocentros constituyen espacios públicos de acceso a internet y a la formación en diferentes tecnologías, pero sus actividades se han reformulado para constituir un agente activo en sus comunidades, por lo que suponen un buen medio para medir el impacto de su papel de intermediación en el empoderamiento ciudadano. Asimismo, son una herramienta que permite la evaluación de la efectividad de las políticas informacionales debido a su número, volumen de usuarios y extensión a los cantones rurales y provincias del país, de manera mucho más activa que las redes de bibliotecas públicas, tras la desaparición del Sistema Nacional de Bibliotecas en, 2014, y su proceso de certificación que ha durado hasta 2016.

Ecuador desde hace unos años ha puesto los cimientos para el desarrollo de la Economía del Conocimiento. A partir del Ilamado Gobierno de Revolución Ciudadana y la Carta Magna de 2008, la educación y el acceso a la cultura sufrieron transformaciones esenciales. Las nuevas líneas están guiadas por el desarrollo programático del Sumak Kawsay o también llamado "Filosofía del Buen Vivir", una nueva visión para conceptualizar el desarrollo económico y social basado en la cosmovisión indígena y que representa una propuesta conceptual y política a partir del equilibrio integral de los procesos sociales, económicos y ambientales (García Álvarez, 2014).

La Economía Digital ha constituido un aspecto clave en los procesos de cambio del país, avanzándose en indicadores estructurales de conectividad, amplitud de la oferta y los servicios basados en TIC (Martínez Cardama y otros, 2015). En este sentido se fija como objetivo prioritario la transformación tecnológica del país con metas concretas en servicios digitales como:

- Alcanzar un índice de digitalización de 41,7. Este índice mide el nivel de adopción de las TIC. En este sentido el plan también trató de incrementar el número de usuarios de estas tecnologías al $50 \%$.

- Alcanzar un índice de gobierno electrónico de 0,65 . Este indicador de Naciones Unidas mide la capacidad de las administraciones públicas de utilización de las TIC en la prestación de servicios.
- Disminuir el analfabetismo digital al 17,9\%, enmarcado dentro de la transformación industrial y tecnológica del país.

Estas metas se han articulado a través de una estrategia específica denominada "Ecuador Digital 2.0"que tiene por objeto mejorar tanto la infraestructura tecnológica como las competencias de la ciudadanía. A través de este plan se articula otro orientado específicamente a proveer servicios tecnológicos universales: el Plan Nacional de Acceso Universal y Alistamiento digital (PLANADI) en sus dos etapas: una primera (PLANADI 1.0), orientada a la introducción de las TIC, y una etapa llamada PLANADI 2.0, conectada a la praxis social de las TIC, con módulos dedicados a los servicios digitales públicos, redes sociales o emprendimiento a través de las TIC. Estas líneas son en las que se centra este estudio, ya que constituyen el medio a través de las cuales se puede realizar una valoración más precisa del estado del empoderamiento digital de la ciudadanía.

\subsection{Objetivos del estudio}

Las políticas informacionales mencionadas y los cambios en el país han sido la razón por la que plantearse una evaluación del papel de los infocentros en la consecución de una adquisición de competencias digitales más reales y verificables. Además, las siguientes cuestiones fueron tenidas en cuenta:

- ¿Cuál es el papel real de los infocentros en la reducción de la de brecha digital?

- ¿Cuál es el grado de implicación real de los infocentros en sus comunidades y en especial, en colectivos vulnerables para el acceso a las TIC como las mujeres de estas zonas rurales, las minorías indígenas y personas con diversidad funcional?

El objetivo fundamental de este estudio es evaluar el desarrollo de servicios llevados a cabo por los infocentros en Ecuador para fomentar el empoderamiento digital en su comunidad. Paralelamente, se pretende conocer los proyectos concretos llevados a cabo dedicados a colectivos específicos con mayor necesidad de adquisición de poder colectivo, como las iniciativas para la lucha contra la desigualdad de género y el empoderamiento de la mujer, colectivos indígenas o la tercera edad. Esta mirada permitirá averiguar las posibles brechas existentes y la existencia de programas especializados para la mejora de la apropiación de las TIC.

Todos estos ámbitos de evaluación, innovadores desde un solo estudio a través de infocentros, permitirán servir de soporte para la toma de decisio- 
nes en la futura modelización de servicios de los infocentros y en su capacidad formativa, ya que detecta necesidades reales de las comunidades donde estos se asientan. Este trabajo, constituye, un análisis cualitativo más profundo al que ofrece en la actualidad el propio Ministerio de Telecomunicaciones (https://infocentros.mintel.gob.ec), ya que no solo recoge datos, sino percepciones de los directores y capacitadores de los infocentros lo que constituye un reflejo de sus inquietudes y problemas diarios y toma el pulso a los proyectos llevados a cabo que promuevan de manera activa el empoderamiento digital.

El presente trabajo es el culmen de un proyecto financiado por la Secretaría de Educación Superior, Ciencia y Tecnología e Innovación de Ecuador (SENESCYT) cuyo fin era la evaluación y prospectiva de la Sociedad de la información en Ecuador a través de los diversos agentes implicados, y que ha tenido como resultados ya publicados, la radiografía de las políticas informacionales del país y propuestas de mejora (Martínez Cardama y otros, 2015) y la evaluación del papel de las bibliotecas universitarias como agente de cambio en el desarrollo de un marco de provisión de servicio de contenido digital (Caridad Sebastián y otros, 2018). Por último, se pretendió verificar la efectividad de las políticas informacionales a través del grado de empoderamiento digital de la ciudadanía.

\section{REVISIÓN DE LA BIBLIOGRAFÍA}

\subsection{Infocentros: desarrollo y estado de la cuestión}

Los infocentros llevan décadas ejerciendo su actividad, y, de acuerdo, con Breitenbach (2013) han sido infravalorados como herramienta fundamental en el desarrollo rural y económico de los países y el empoderamiento de sus usuarios a nivel socioeconómico. El abaratamiento del precio de internet en los hogares, ligado a su extensión; junto con la telefonía móvil y el auge de la autoformación en línea ha desvirtuado estos centros comunitarios que deben de reivindicarse ante los gobiernos y políticas informacionales como grandes espacios de conocimiento compartido.

Su aparición tiene lugar a medidos de los 80 en las zonas rurales de países escandinavos y su desarrollo en Europa está muy ligado a las políticas de la Unión Europea de la década de los 90 que trataba de garantizar puntos de acceso público a Internet cohesionando especialmente a las distintas áreas rurales de los países miembros. Así, fueron objeto de financiación en planes como Acción E Europe (2002) o 2010 que tenían como objetivo la inclusión digital y el papel de la tecnología en la mejora de la educación, la sanidad y el desarrollo social.

No existe una unificación terminológica en la denominación de estos centros, así, Colle y Roman (1999) y Lancaster (1999), identificaron en sus estudios más de 30 denominaciones entre las que destacan, en lengua inglesa: Telecentre, Telecottage, Community Teleservice Centre (CTSC) o Community Technology Centre.

Lo mismo sucede con su definición, comúnmente delimitada a espacios físicos que proveen servicios tecnológicos, generalmente para fines educaciones, sociales o económicos vinculándose a una comunidad local. Aunque su identificación como espacio físico pueda ser sencilla, su provisión de servicios a lo largo de las décadas ha cambiado. Aparecieron ligados al teletrabajo (Meshur, 2012) pero a la vez pueden ser una fuente en sí misma de empleo, y una solución puente para los emprendedores, autónomos y pymes. El espacio se transformaría así en una incubadora de proyectos, adoptando fórmulas novedosas que fomenten el coworking y emprendimiento especialmente en un marco de crisis.

El desarrollo de los infocentros ha estado vinculado siempre a políticas públicas como un argumento para el fin de la exclusión digital en zonas rurales (Benjamin y Dahms, 1999, Breitenbach, 2013). No obstante, desde la década de los 90 , la situación cambia, $y$, aunque muchas redes de infocentros se han reconvertido en modelos más integradores y flexibles, sin ayudas públicas, estos han luchado por sobrevivir y su sostenibilidad ha sido debatida desde tres perspectivas: la económica, la política y la social (Bailur, 2007, Masiero, 2011). En cuanto a las dos primeras, están marcadas por la dependencia de los recursos públicos y la voluntad política de continuar con estos centros. Esta justificación es en la que se apoya buena parte de la bibliografía, ocupándose más de los asuntos organizacionales de estos centros y sus servicios, que de su implicación real en todas las capas de la sociedad (Kumar y Best, 2006), y en cuanto a los estudios sobre su impacto, este casi siempre se ha valorado desde una perspectiva económica.

Existe escasa bibliografía sobre su apropiación social y capacidad de influir en la comunidad y stakeholders locales, adaptándose a las nuevas demandas sociales. Este aspecto, fue incluido de manera somera en la metodología de indicadores detallada por Morales García y otros (2009) bajo el epígrafe de "relación con otros organismos", aunque solo de manera cuantitativa, midiendo el número de contratos o convenios con empresas e 
instituciones locales. Asimismo, se ponía el foco en la colaboración con bibliotecas públicas, dada la transversalidad existente de ciertos servicios en zonas donde una biblioteca pública no está presente. No obstante, es necesario incidir en un mayor análisis social de la comunidad y, sobre todo, en los procesos de empoderamiento que surgen en torno a los infocentros.

En la actualidad, la investigación académica más reciente versa sobre estudios de caso en países en vías de desarrollo y su aplicación al entorno rural (Furuholt y Sæbø, 2018, Lwoga y Chigona, 2018), así como investigaciones por colectividades en riesgo de exclusión digital, como el caso de la tercera edad, o la aplicación y medición concreta de políticas digitales nacionales, como el caso de Brasil (Brito y otros, 2018).

En el caso de Ecuador, este trabajo adquiere especial relevancia ya que apenas existen aproximaciones al respecto. Esta situación, es similar a la de otras estructuras de conocimiento como las redes de bibliotecas. En cuanto a estas, se constata la ausencia de literatura especializada, tal y como reconocen Álvarez Muñoz y Hernández Domínguez (2016), que la caracterizan por su generalidad y carácter histórico. Las principales aproximaciones actuales se centran en ámbitos como la Bibliometría, o las bibliotecas universitarias, especialmente a partir de la acreditación de las universidades en Ecuador que ha constituido una oportunidad y un reto a nivel de investigación (Molina Benavides, 2015, Álvarez Muñoz y Hernández Domínguez, 2016, Caridad Sebastián y otros, 2018).

Asimismo, existe una ausencia de información cuantitativa o indicadores de medición sobre estas instituciones. Lo mismo sucede con las unidades escogidas para esta investigación, los infocentros, de los que se dispone solo de información factual y descriptiva o, principalmente, sobre el estado de la conectividad en comunidades rurales (Calderón y Acuña, 2017). El presente artículo supone un paso más en un estudio de esta realidad como lugar de empoderamiento y formación de masa crítica ciudadana mediante la percepción de los propios formadores sobre su impacto social.

\subsection{Concepto de empoderamiento: Origen, definición y aplicación en el estudio}

El concepto de "empoderamiento" siempre se asocia a la reivindicación de grupos vulnerables en reafirmar su situación dentro de una colectividad. A pesar de este componente social, el empoderamiento puede ser contemplado como un proceso individual de "transformación" por el cual el ciudadano adquiere poder y control para la toma de decisiones y la conquista de objetivos que le son propios. El Diccionario Panhispánico de Dudas, dio respuesta al uso del término en español, delimitándolo a "la concesión de poder [a un colectivo desfavorecido socioeconómicamente] para que, mediante su autogestión, mejore sus condiciones de vida". Constituye un concepto transversal que puede tratarse desde diferentes perspectivas: económica, social, educativa...y, en definitiva, desde cualquier aspecto que implique una toma de conciencia del individuo como agente empoderado con igualdad de oportunidades y capacidad de influencia en la toma de decisiones.

El término en el contexto digital, el concepto de "empoderamiento digital" no se concibe como una mera consecuencia del uso de las tecnologías por la ciudadanía sino como la capacidad de ser incluyente en la sociedad a partir de unas competencias adquiridas a partir de su uso (Mäkinen, 2006), extrayendo su máximo potencial en el ámbito económico, legal o técnico (Kirti y Dipak, 2016). El empoderamiento digital entronca con los nuevos espacios dinámicos que habilita la tecnología en la actual Sociedad de la Información, por lo que está altamente ligado con los conceptos de Multialfabetización digital, y la capacidad para decodificar de manera crítica la información e interactuar con ella (Fuente-Cobo, 2017).

La red de infocentros del país constituye la institución clave, en importancia geográfica y social, para evaluar este tipo de competencias debido a la amplitud y extensión por todo el territorio ecuatoriano, cubriendo zonas rurales y vulnerables donde otros agentes sociales como la biblioteca pública en ocasiones no tiene presencia. Es importante evaluar el grado de apropiación e integración de estas tecnologías en estas comunidades y analizar los procesos de empoderamiento y liderazgo que de ellos deriven. Asimismo, en 2018 se está produciendo un reconocimiento de los infocentros como facilitadores de la Universidad Virtual del país. Este convenio a nivel formativo refuerza la presencia de la red de infocentros y prevé que su papel sea aún más importante en el futuro próximo.

\section{MÉTODO Y MATERIALES EMPLEADOS}

El estudio se basa en los datos proporcionados por los propios responsables de los infocentros. El universo de infocentros está formada por 854 infocentros y megainfocentros. La diferencia entre ambos es infraestructural (mayor capacidad de salas y equipamiento y de formadores). Los infocentros tienen una cobertura del $78 \%$ del total de cantones rurales del país, mientras que los megainfocentros cubren regiones de mayor concentración poblacional. 
Los datos de localización se obtuvieron gracias a la colaboración con el Ministerio de Telecomunicaciones y Sociedad de la Información del país. Esta institución facilitó a los investigadores de este proyecto una base de datos con información de contacto de 854 infocentros. Tras su análisis, se redujo la muestra a 829 centros, debido a que 25 no poseían conectividad a internet, o estaban todavía en proceso de encontrar un facilitador o profesional encargado del centro. Debido al alto número de participantes, el método elegido para obtener los datos de interés fue la realización de un cuestionario ad-hoc a través de Google Forms, que fue enviado junto con una carta de explicación y apoyo del proyecto, en la que se explicaba el objeto de la investigación, así como el tratamiento anónimo de las respuestas. La encuesta ha estado disponible desde diciembre 2016 hasta enero de 2018.

Para la elaboración de las preguntas, especialmente en el apartado de servicios, se han tenido en cuenta las líneas marcadas por proyectos como ELAC2020 (Agenda Digital para América Latina y el Caribe) elaborado por CAF (Banco de DesarroIlo de América Latina, 2013), a nivel europeo la reformulación hacia indicadores de mayor dimensión informacional como los que marca The Digital Competence Framework 2.0 (Comisión Europea, 2016), y en las líneas estratégicas marcadas por el Ministerio de Telecomunicaciones de Ecuador, para la reducción de la brecha digital, cuyo análisis ha desembocado en el reciente Libro Blanco de la Sociedad de la Información (Ministerio de Telecomunicaciones de Ecuador, 2018).

Constituyó un cuestionario mixto y autoadministrado, que incluyó preguntas abiertas y cerradas.
En lo que respecta a estas últimas se eligieron los formatos de respuesta múltiple que permitían varias respuestas y se adoptó la escala Likert para algunas referidas a valorar el grado de asiduidad de los visitantes a los centros. Las dimensiones y estructura del cuestionario la componen tres bloques temáticos o secciones principales. La primera sección identificaba a los infocentros, el volumen y perfiles de asistencia por colectivos, el número de empleados y su formación y los problemas cotidianos que como empleados suelen hacer frente. La segunda se centró en los modelos de servicios ofrecidos, tipo y temática de la formación impartida y la colaboración existente con los agentes locales. La última parte recoge las respuestas relacionadas con los programas específicos de empoderamiento digital y trata de conocer su existencia, perspectiva y a qué colectivo se aplican.

\section{RESULTADOS Y DISCUSIÓN}

Una vez finalizado el plazo para participar en esta investigación, se contó con la perspectiva de 297 infocentros, lo que supone una tasa de participación del $35,8 \%$. Se muestran a continuación los principales resultados del estudio estructurados en las tres áreas de interés que conforman el cuestionario:

\subsection{Datos generales}

En cuanto a la adscripción de los infocentros que han respondido, 22 de las 24 provincias están representadas en este trabajo. Se han obtenido respuesta de infocentros adscritos a todas las provincias del país, salvo Los Ríos (Costa) y Galápagos (región insular que carece de infocentros) (Figura 1).

Figura 1 Distribución de respuestas por cada provincia de Ecuador

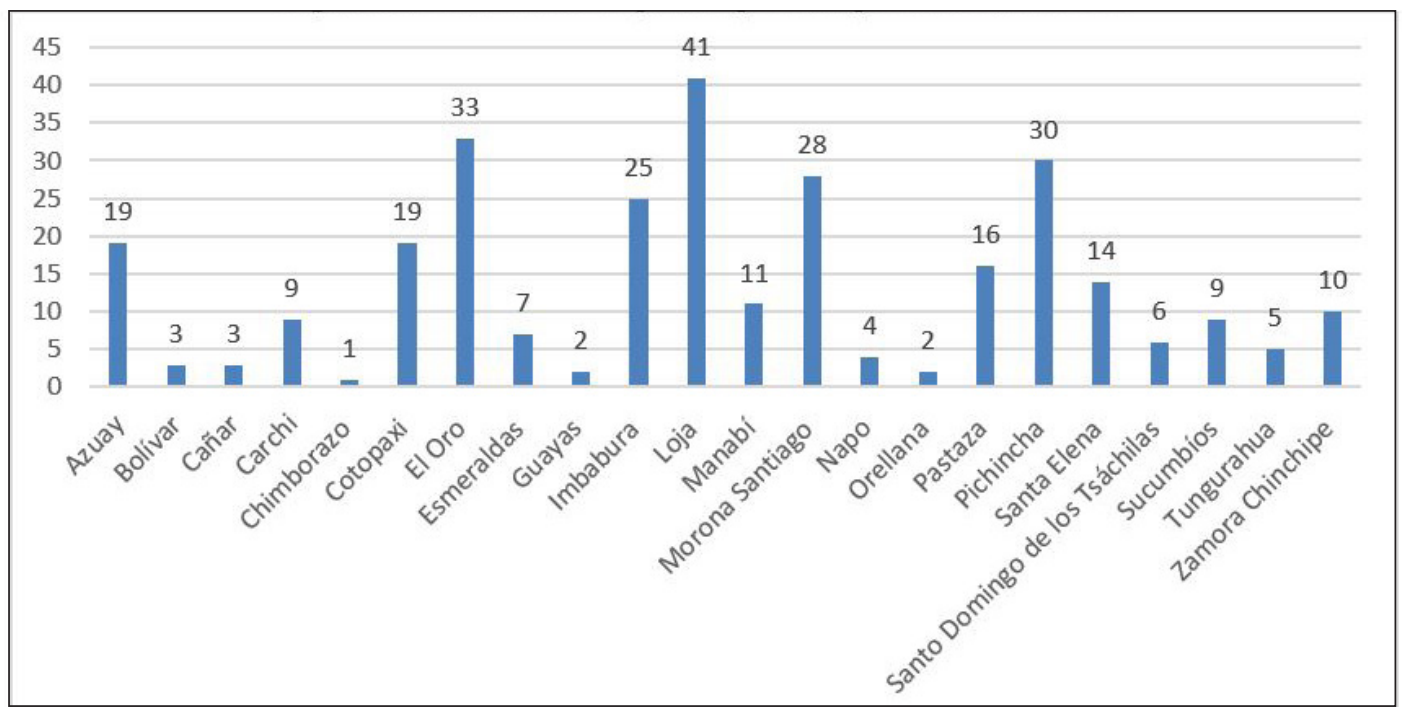


De estas respuestas, según su estructura, se contó con la respuesta de 290 infocentros y 7 megainfocentros. Llama la atención que el $85.5 \%$ de los infocentros participantes se sitúan en zonas y parroquias rurales del país; algo que, sin duda, marca la dinámica de respuestas posteriores en temas de dotación tecnológica o conectividad, a diferencia de centros en sitios urbanos.

El 96.6\% asegura llevar un registro de asistentes. En cuanto a su número, el promedio de todos los registros es de 4916 usuarios en un año, lo que suma un total de 1.460 .052 usuarios del total de los infocentros que han respondido. Son datos que pueden considerarse como relevantes, ya que, según el Ministerio de Telecomunicaciones, la red total de infocentros cubre las necesidades de 6.2 millones de personas

El target que con mayor asiduidad visita el centro de manera diaria son los adolescentes y el público infantil (Figura 2).

Su frecuencia diaria es de $65.4 \%$, mientras que en los adolescentes esta crece a un $71.3 \%$. Los siguientes grupos muestran perfiles de asistencia desigual, cayendo la frecuencia diaria a moderada u ocasional como en el caso del colectivo de la tercera edad y el de personas con discapacidad. Los perfiles relacionados con el entorno laboral se han dividido de manera expresa en aquellos por cuenta propia, asalariados y desempleados, con el fin de cotejar posteriormente si los programas atiendan necesidades de emprendimiento, reciclaje profesional o a la creación de proyectos. La presencia de este colectivo se divide entre ocasional y frecuente, por lo que es necesario que se tenga en cuenta a la hora de diseñar el contenido de la formación de los infocentros. Sin embargo, sin duda, es en las primeras etapas (infancia y adolescencia) donde los infocentros son más utilizados. Esto puede manifestar una visión infraestructural del mismo: solo contemplado como lugar para el acceso a Internet o para el estudio y apoyo a la docencia en las primeras etapas.

En lo que respecta al número de empleados, 229 infocentros contestaron que solo disponen de un empleador, frente a 7 que respondieron que 2, que corresponden con los megainfocentros cuyas dimensiones requieren de mayor personal. La dispersión geográfica, sumada a la falta de personal en relación a la población que atienden puede ser un factor clave en el mayor o menor éxito de los programas y su ejecución. La formación de los formadores es variada: desde bachillerato hasta técnicos pasando por estudiantes o licenciados universitarios. El requisito mínimo es tener bachillerato, formación más frecuente entre los encuestados. No obstante, la mayoría de las respuestas aluden a que es necesario poder verificar competencias TIC para el acceso a ese puesto. Asimismo, 14 de los encuestados mencionan de manera expresa la capacitación nacional oficial obtenida por el Ministerio de Telecomunicaciones titulada "Auxiliar en Gestión

Figura 2 Perfiles de usuarios y asistencia a los infocentros ecuatorianos

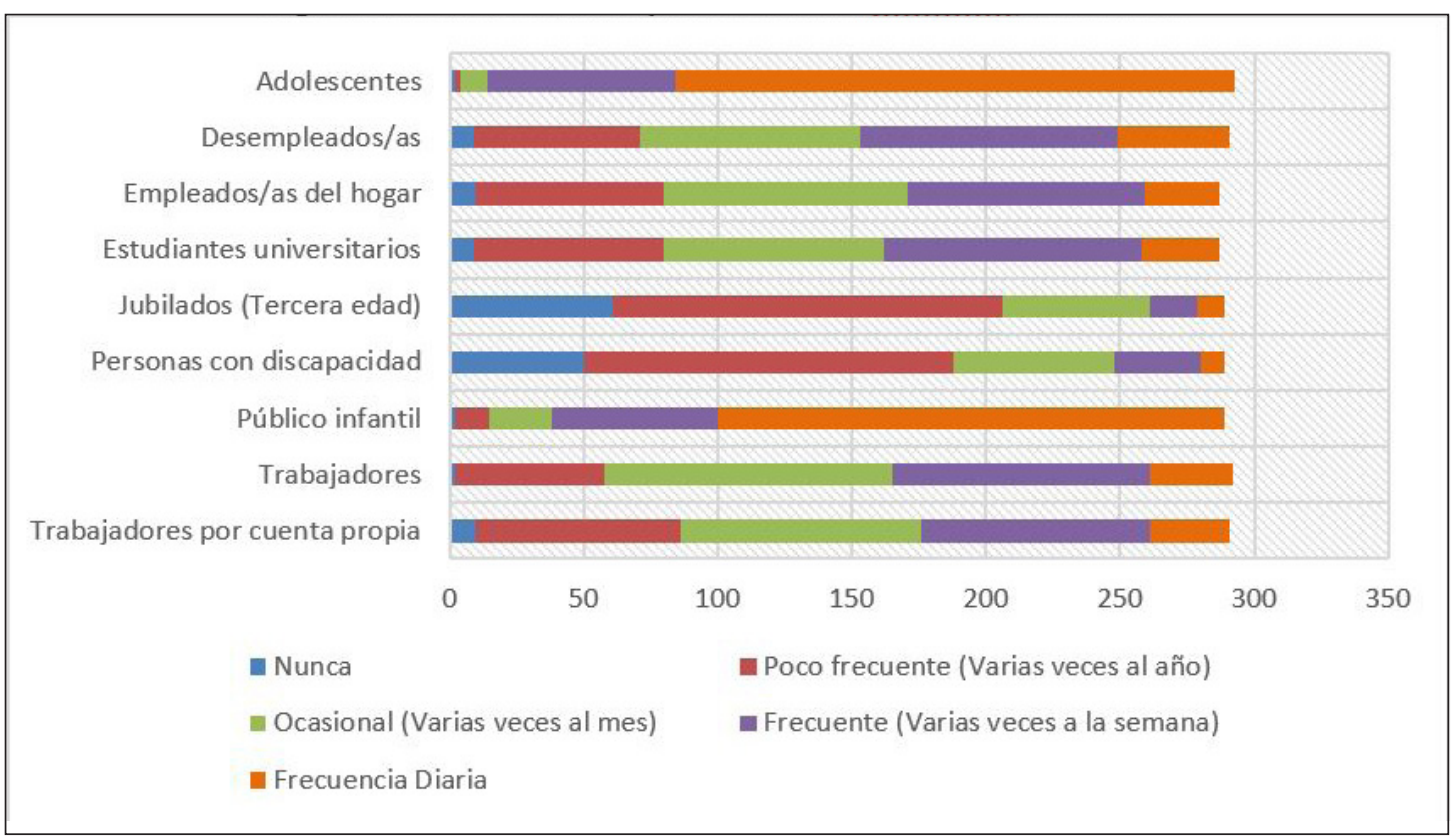


Administrativa y Manejo de TIC" que habilita para trabajar en estos centros. La presencia de este curso legitima el papel de los trabajadores de los infocentros, y nivela las competencias requeridas por los formadores, por lo que es recomendable que en un futuro se extienda a los futuros empleados de los infocentros para obtener una mayor uniformidad en la provisión de servicios.

Sobre los problemas de los formadores en su actividad diaria, las 100 respuestas recibidas en la pregunta abierta muestran que buena parte de las preocupaciones en concreto 32 , se refieren a la poca velocidad de las conexiones VSAT, específico para zonas rurales, problemas de corte de suministro o energía. Asimismo, se menciona la situación deficiente de las infraestructuras de los locales y equipamiento y se cuestiona su validez para ofrecer las capacitaciones de manera adecuada. Otra línea de problemas que ha sido detectada es la falta de apoyo por parte de las autoridades locales, en concreto de los Gobiernos Autónomos Descentralizados (GAD). En cuanto a la participación por parte de la comunidad se menciona el desconocimiento de la población sobre el papel de los infocentros en ofrecer capacitaciones y la poca percepción de utilidad de éstos. Los resultados muestran que todavía existe en muchos usuarios una perspectiva infraestructural sobre estos centros, en los que se piensa que solo proveen acceso a internet. La generalización de internet en los hogares y dispositivos móviles frena, por tanto, la percepción de utilidad. Sin duda, es necesario a tenor de estos resultados, difundir la apropiación competencial de las TIC que los infocentros facilitan.

\subsection{Modelos de servicios de Infocentros}

En primer lugar, se quiso conocer las acciones formativas que apoyan los distintos niveles formativos ecuatorianos. Esto es relevante, ya que desde 2018 se reconoce la Universidad Virtual y el papel de los infocentros como infraestructura de apoyo. En su mayoría, el $78.4 \%$ ofrecen cursos y seminarios solo de tipo presencial, siendo utilizadas las plataformas e-learning en un $48.8 \%$ de los casos. Esto todavía supone un reto no solo a nivel formativo sino infraestructural, debido a los problemas de conectividad encontrados que dificultan el seguimiento de una oferta formativa completamente en línea.

El apoyo a la formación reglada, tanto universitaria como preuniversitaria está presente en el 30,6 $\%$ y $38,7 \%$ respectivamente. Asimismo, el $37.3 \%$ ofrecen servicios de orientación educativa (becas, información institucional). La presencia de público infantil y juvenil favorece que mucha de la oferta formativa se realice desde una perspectiva más informal, en concreto utilizando juegos (32,6\%). Estos resultados muestran que la oferta formativa recoge los cauces formales de las capacitaciones ofrecidas por el Ministerio en cuanto a formación continua, pero que buena parte de los servicios de apoyo se dan a estudiantes para el desarrollo de sus estudios. El hecho de no contar con biblioteca pública en muchos lugares apoya estos hallazgos (Figura 3).

Una de las acciones clave para el desarrollo de la Sociedad de la Información y el Conocimiento en Ecuador, en los últimos diez años, es la apuesta por

Figura 3 Servicios que apoyan acciones formativas en los infocentros de Ecuador

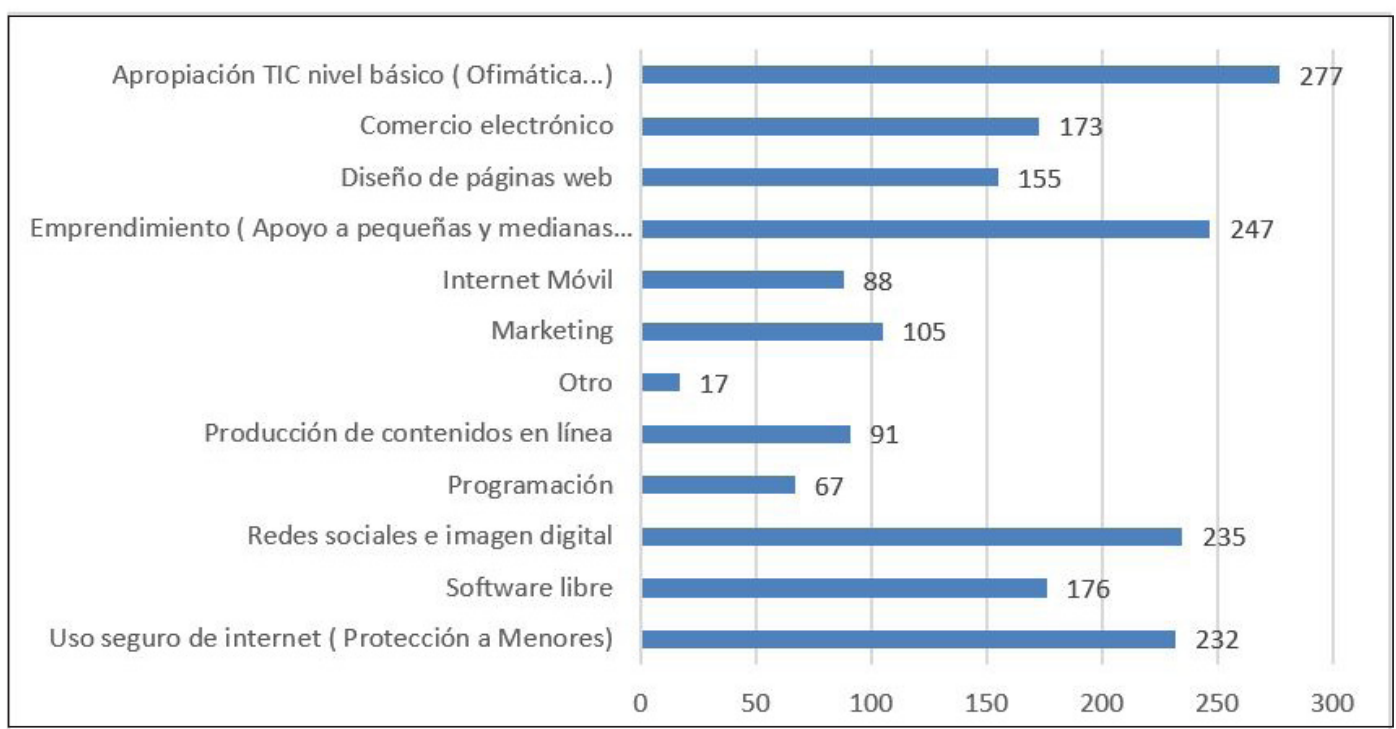


el gobierno electrónico y la mejora cualitativa de la interacción con las instituciones por parte de la ciudadanía. Por tanto, se quiso averiguar cómo influyen los infocentros en la mejora de este indicador, qué tipo de trámites se realizan y cómo es la labor del infocentro como intermediario en la capacitación del usuario. Los resultados muestran que la resolución de trámites administrativos ocupa el mayor nicho de actividad (90.6\%). Se percibieron grandes índices de satisfacción por parte de los usuarios, ya que estas competencias evitan su desplazamiento a otras urbes para realizar trámites de manera presencial. Por otra parte, el fomento de la participación ciudadana, a través de cauces institucionales como foros, consejos ciudadanos sectoriales, asambleas ciudadanas, participación en proyectos concretos como "Presupuestos participativos" se da en un 60,3\%. Ya de manera más minoritaria nos encontramos con asesoramientos de otro tipo como las peticiones de transparencia a las instituciones (17.8\%) y en materia jurídica (9.1\%). Si bien Ecuador posee buenos resultados en materia de apertura de datos (Open Data), siendo el país número 31 de 173 en el Open Data Inventory (2016), la interacción con los mismos es todavía minoritaria, así como el fomento de la transparencia. Los infocentros son un buen eslabón en la mejora de la cadena de gobierno abierto, debido a su cercanía con todas las capas de la ciudadanía.

Para intentar mejorar este servicio, se preguntó en qué temas o servicios institucionales los infocentros ofrecían apoyo o asesoramiento, entre ellos destacan dos fundamentalmente: el asesoramiento en materia de e-Salud (gestión de citas médicas, consulta de historial) $(63,6 \%)$ y la búsqueda de empleo (68,3\%). Ambas son dos tendencias en las que los infocentros han sido pioneros a nivel internacional. En cuanto a la primera, debe extenderse más allá de la capacidad de realizar estos trámites on line a la formación, por ejemplo, en herramientas (apps) que permiten una mejora de la vida de los pacientes, la concienciación sobre la importancia de adoptar hábitos como la alimentación saludable, información sobre trastornos alimenticios, etc. Por su parte, como soporte a la búsqueda de empleo, el infocentro debe asesorar al ciudadano sobre qué páginas o recursos utilizar para obtener resultados en línea (bien a través de recursos institucionales u otros) y proporcionar formación adecuada para este fin. Por último, la realización de trámites bancarios ocupa también $n$ un lugar elevado (60.9\%). Nuevamente, la percepción de utilidad de estas respuestas se encuentra presente, ya que estos trámites ayudan a mejorar la vida cotidiana de los individuos a efectos de movilidad geográfica.

En cuanto a las capacitaciones en materia TIC, aunque buena parte de las líneas de los planes PLANADI 2.0 versan sobre su aplicación a todos los ámbitos, se quiso ver la frecuencia y la oferta ofrecida en la red durante el periodo del estudio (Figura 4).

Figura 4 Oferta de capacitaciones en materia TIC de los infocentros ecuatorianos

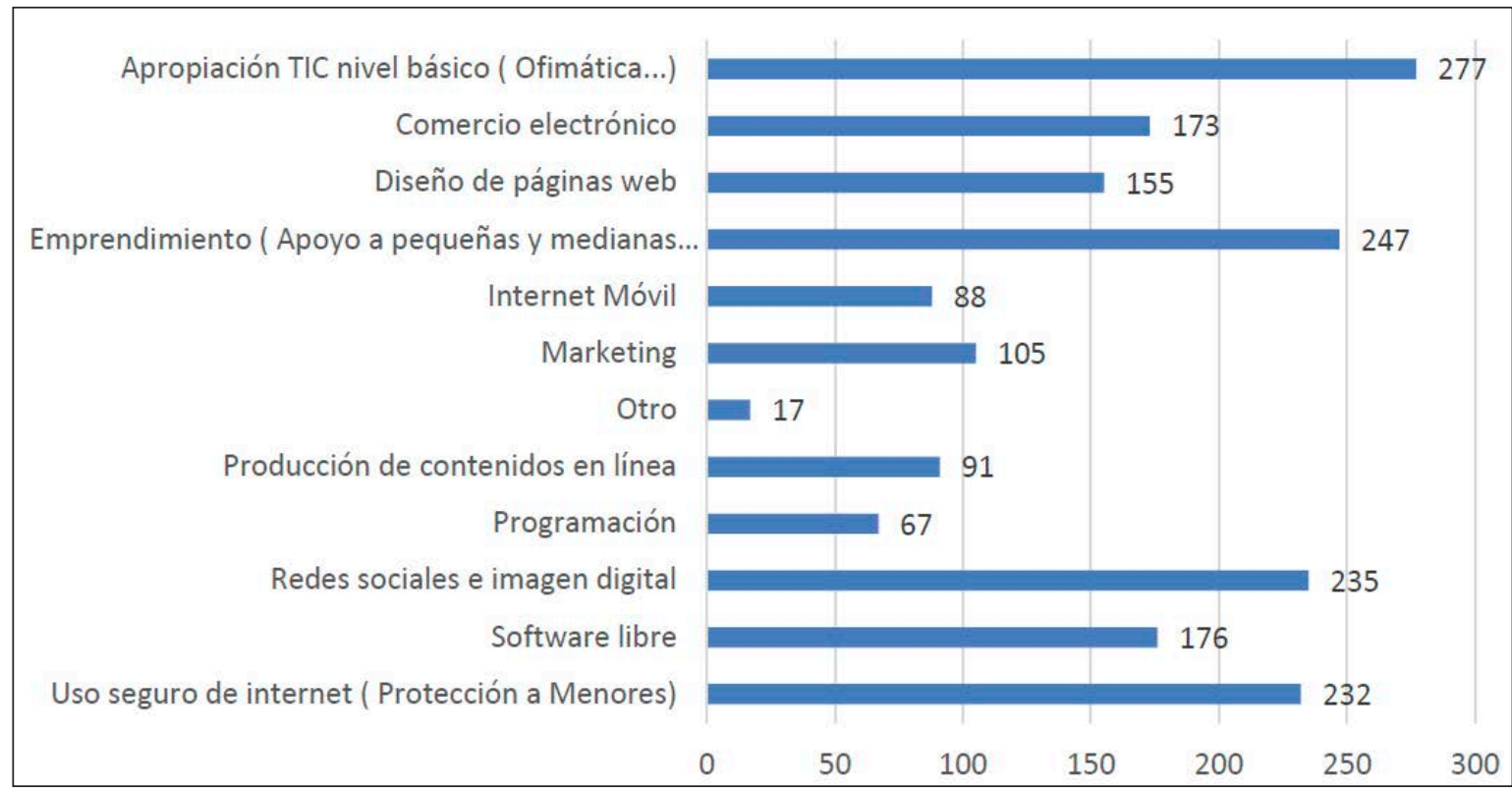


La oferta reside en la formación en Ofimática y la iniciación a una informática básica (96.5\%), El emprendimiento y el asesoramiento a empresas ocupa un lugar destacado, con un $86.1 \%$. Es abundante también la formación en redes sociales e imagen digital (79.1\%) y los aspectos relacionados con seguridad, en especial en el uso de Internet por parte de los menores (78.1\%). La formación en otras competencias como desarrollo web, software libre o marketing digital también goza de buenos resultados.

La participación en el espacio local es clave para la viabilidad de los infocentros, y que estos tengan un mayor calado en la comunidad. Esta, no se logra de manera aislada, sino en colaboración con todos los agentes locales. No obstante, a tenor de los resultados, es todavía muy escasa. Sorprende que el $65,5 \%$ responda que no colabora con la biblioteca pública en caso de que la haya. Las líneas en las que se formula la colaboración están muy lejos de ser adecuadas: muchos solo comparten instalaciones y colección. Otra de las respuestas, de manera aislada, sí reconoce la organización de cursos y talleres conjuntos por ambas instituciones. Se observa en muchas ocasiones que el infocentro trata de cubrir el papel de la biblioteca cuando esta no existe, algo frecuente a tenor de muchas respuestas. En este sentido, el infocentro se plantea como punto de acceso y asesoramiento a las bibliotecas digitales del país, apoyando los procesos formativos de los estudiantes mediante tareas de selección de fuentes fiables para la investigación.

\subsection{Empoderamiento digital: Programas es- pecíficos}

La identificación de proyectos concretos que apoyen el empoderamiento ciudadano es clave para conocer si estos infocentros son agentes de cambio social y poder proponer líneas de acción futuras. En primer lugar, se consideró esencial conocer la perspectiva del empoderamiento en las acciones llevadas a cabo. Los resultados muestran que el $65,9 \%$ de los infocentros cubren acciones relacionadas con la consecución de mayor autonomía en el entorno laboral. Esto lo demuestra la oferta de capacitaciones existente, con clara vocación a la incorporación al mercado laboral, reciclaje profesional o adquisición de nuevas competencias en este entorno. En segundo lugar, los formadores consideran que el empoderamiento personal (toma de decisiones, autoestima...) está presente en el $50,1 \%$ de las actividades. Llama la atención que el empoderamiento femenino o de la mujer solo se destaque en el $16,1 \%$ de las respuestas. Esto es sorprendente, ya que muchos proyectos tie- nen como destinatario este colectivo mediante la puesta marcha de actividades concretas como la comercialización de productos o la creación de microempresas que sí debieran ser elementos facilitadores de su empoderamiento y autonomía.

Centrándonos en este colectivo, interesó ver en qué medida las mujeres participan en las comunidades en las que el infocentro atiende. La formación, es la opción más señalada cuando se pregunta por las necesidades que el infocentro puede cubrir a las mujeres de la zona, y destaca especialmente su capacitación en TIC de manera elemental, a nivel usuario (76\%). En cuanto a la aplicación de esta formación y el contexto en que se desarrolla, algunos formadores señalan cómo las mujeres acuden a los centros a aprender a consultar el expediente escolar de sus hijos en la escuela a través de Internet, y aprender competencias informacionales para poder ayudarles en sus tareas. Por tanto, puede verse que sí existe un trasvase de ese conocimiento al entorno familiar. En cuanto al laboral, la necesidad que cubre el infocentro para el empoderamiento de las mujeres es la capacidad de favorecer su emprendimiento (75\%). Dado que no existen acciones en las que el empoderamiento femenino sea un elemento nuclear, los cursos de emprendimiento a nivel general dificultan la puesta en marcha de iniciativas para un colectivo vulnerable como el de las mujeres de zonas rurales, con menor formación, más cargas a nivel familiar y menor capacidad de acceso al crédito. Es precisamente en estas zonas rurales, donde las acciones de empoderamiento femenino por parte de los infocentros han tenido más efecto en el incremento de la formación e ingresos, a pesar de barreras infraestructurales asociadas a la falta de conectividad de los centros, situación muy común en la literatura consultada (Lwoga y Chigona, 2018). En relación a este estudio, las áreas donde los responsables de los infocentros perciben mayor utilidad en el colectivo femenino son la búsqueda de empleo (53.5\%) y la necesidad de mayor autonomía individual en su entorno (36\%). La falta de programas con enfoque de género muestra que solo el $37.9 \%$ reconoce que los infocentros promueven el empoderamiento real de la mujer en un entorno laboral. Asimismo, las actividades que tengan como tema principal o transversal la prevención contra la violencia de género son residuales $(15,4 \%)$. Por último, los facilitadores también reconocen la escasa sensibilidad hacia la perspectiva de género por parte de las directrices de la coordinación de los Infocentros (14.7\%). El empoderamiento femenino se fundamenta en la superación de los procesos de desigualdad de género, por tanto, es un elemento clave para el posicionamiento de las mujeres en la sociedad. Los infocentros han de replicar y reproducir las políticas de igualdad de género del país y ser capaces de marcar esta impronta en proyectos a nivel local. Los resultados de 
este estudio todavía avalan la tendencia detectada en la investigación de la falta de un enfoque de género en la planificación de las actividades de estos centros (Alao y otros, 2017).

En cuanto al análisis de la situación de las minorías indígenas, cabe señalar que Ecuador cuenta con aproximadamente un millón de indígenas, que representan un $7 \%$ de la población (CEPAL, 2014). Aunque el número de población nativa es importante, el $86.8 \%$ de los infocentros no disponen de iniciativas para este colectivo, ya que, como señalan los formadores, o bien no se encuentran en su parroquia o no son usuarios de los infocentros. De entre los proyectos concretos encontrados y sobre los que se pide información, se pone el foco de manera especial en la necesaria formación en TIC de este colectivo debido a las barreras todavía existentes. Asimismo, otras iniciativas dignas de mención versan sobre la formación y aprendizaje de la comunidad local de su lengua para favorecer la relación intercultural. La mejora de sistemas tradicionales de fabricación constituye otra de las acciones clave.

La capacidad de apoyo a personas con diversidad funcional se cifra solo en un $27.9 \%$. El $67.3 \%$ reconoce que el infocentro es accesible. Entre los tipos de adaptaciones las más comunes son la adaptación de equipos (42.7\%), la superación de barreras arquitectónicas $(23.2 \%)$ y, en menor medida, los programas específicos de discapacidad (9\%). No existen de manera clara iniciativas en las capacitaciones oficiales para este colectivo necesarias para llevar a cabo una política inclusiva digital.

Con el fin de ejemplificar las respuestas, se solicitó a los formadores proyectos concretos que hayan surgido a partir del infocentro y que apoyen el empoderamiento de las comunidades a las que sirven. Estas respuestas se sistematizaron mediante un sistema de categorías creadas ad hoc (Tabla I).

Esta categorización corresponde a iniciativas concretas que los formadores han desarrollado en sus centros y promueven el empoderamiento ciudadano. A través de ellas, se pueden detectar algunas perspectivas. La primera, la validación del infocentro como espacio institucional adecuado para la participación pública. Esto viene refrendado no solo por las capacitaciones oficiales que guían la formación en estos centros, sino por los convenios instituciones como los desarrollados por el Ministerio de Agricultura o por el desaparecido, Ministerio Coordinador de Conocimiento y Talento Humano. Existe, por tanto, una formalización en el apoyo local a decisiones o políticas gubernamentales que ha de ser cuidada y mejorada a efectos de comunicación y coordinación.
De manera paralela a la acción institucional, a nivel de desarrollo del capital social se percibe un segundo plano de interés que los infocentros han de fomentar más y que es el que verdaderamente permite un empoderamiento real: la creación, mejora y control sobre recursos y activos propios y comunitarios (puede verse en el caso de la creación de bancos de microcrédito). Esto también implica el aprovechamiento de los espacios públicos en consonancia con varios actores de la comunidad. Por último, en las acciones analizadas puede verse la importancia de la organización como fórmula de acción, es decir, personas que perteneciendo a un sector social excluido puedan participar de manera colectiva en la comunidad, reforzando y ampliando su visibilidad.

No obstante, los proyectos de empoderamiento destinados a colectivos específicos tienen una representación testimonial en la totalidad de la red. La ausencia de iniciativas oficiales que tengan como target estos colectivos, dejan al abur del voluntarismo y la creatividad de los responsables de los infocentros la ejecución de estos proyectos. Es necesario, teniendo en cuenta la experiencia de las capacitaciones durante estos años, una evaluación de las mismas incluyendo la representación de estos colectivos en la que deben colaborar asociaciones y actuales participantes de proyectos.

Asimismo, debe realizarse un plan de seguimiento y evaluación de todas las iniciativas planteadas, especialmente aquellas ligadas al emprendimiento, que tienen más riesgo de desaparecer a corto plazo. La falta de recursos, formación empresarial y acceso a mercados competitivos han de paliarse a través de asesoramiento específico durante toda la duración del proyecto empresarial, no solo al inicio.

\section{CONCLUSIONES}

Los resultados de esta investigación ofrecen importantes hallazgos sobre la situación de las medidas institucionales para la reducción de la brecha digital y el fomento de la inclusión digital en Ecuador. Los infocentros constituyeron los agentes adecuados para evaluar estas políticas, debido a su amplia presencia territorial y el volumen y tipología de población a la que atienden.

1. En primer lugar, la investigación muestra una relación positiva entre las líneas clave a nivel institucional para el fomento de la Sociedad de la información y las acciones de los infocentros, ya que las capacitaciones formales ofrecidas cubren buena parte de aspectos cubiertos en el Plan Nacional de Alistamiento Digital (TIC a nivel medio y avanzado, Marketing Digital, Turismo 2.0, emprendimiento...). 
Tabla I. Proyectos concretos de los infocentros ecuatorianos que apoyan el empoderamiento de las comunidades

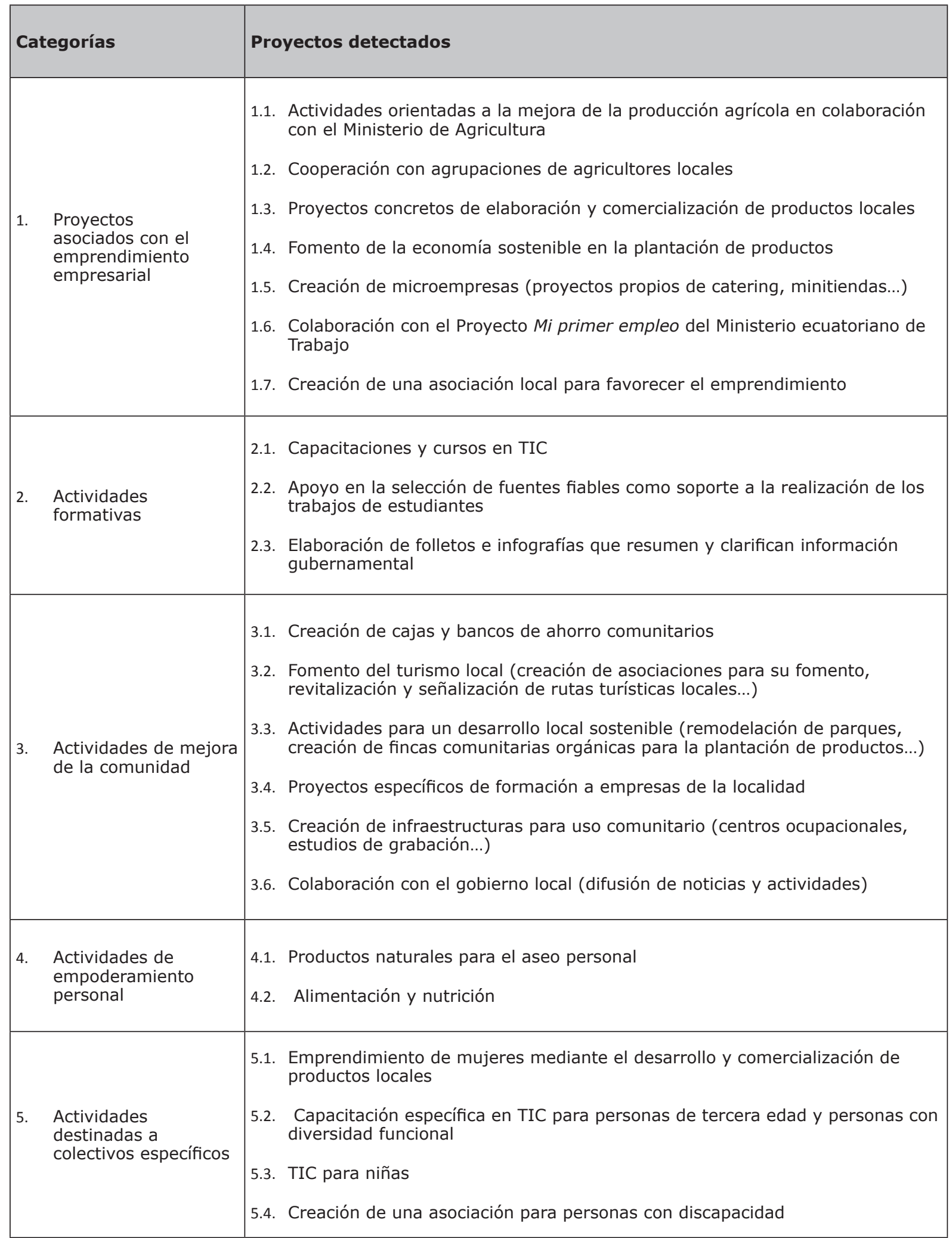


2. La metodología del estudio basado en la elaboración de un cuestionario autoadmistrado a los formadores y facilitadores presenta algunas limitaciones en cuanto a la interpretación y generalización de los resultados, ya que no contempla la opinión directa de los usuarios. Sin embargo, la opinión y respuestas de los formadores han permitido la realización de un estudio a fondo de la realidad de los infocentros en Ecuador a través del cual se han obtenido valiosos resultados.

3. En referencia a la tipología de asistentes, el estudio señala como el público infantil y juvenil es el que visita estos centros con más frecuencia. Los formadores apuntan al papel de la red como apoyo en la elaboración de trabajos de investigación de los estudiantes y la selección de fuentes de información fiables. Este hecho implica que el infocentro suple, $y$, en algunos casos, surge de la reformulación de la biblioteca pública. Aunque la certificación nacional de los gestores de los infocentros es en sí misma un hito, esta debe ir más allá del propio manejo de las herramientas hacia su uso crítico mediante la adquisición de unas competencias más amplias y transversales que implicquen una verdadera alfabetización digital, como la búsqueda y evaluación de la fiabilidad de la información, así como su decodificación crítica. La formación en estas competencias informacionales requiere de profesionales de la información y documentación, cuyo papel debe estar más presente en el diseño de la oferta formativa.

4. Los problemas ligados a la infraestructura local y la conectividad se han manifestado cómo uno de los principales hándicaps hallados para la puesta en marcha efectiva de proyectos. La mejora de la conectividad es un elemento transversal de mejora en todos los ítems evaluados, ya que posibilita formación ofrecida por el centro, y de manera muy especial, la capacidad que ha de tener el centro como apoyo al e-learning, y especialmente al reto que supone desde 2018 las 30.000 plazas de formación virtual en las universidades del país.

5. Otro de los retos futuros para estos centros es el incremento de la percepción de utilidad por parte de la ciudadanía. Es destacable que los servicios más valorados son aquellos que revierten de manera directa en la vida de los individuos (gobierno electrónico, gestión de e-salud). Asimismo, los formadores reconocen que la labor de los infocentros repercute de manera positiva en las salidas laborales de los asistentes, por lo que, en materia de retorno de inversión, existen evidencias rigurosas del papel de los infocentros en re- ducción de la brecha digital y la mejora de la vida de los ciudadanos.

6. Otro factor clave para su supervivencia es la falta de colaboración con los diversos agentes locales de las poblaciones en las que se asientan. El empoderamiento ciudadano, requiere de participación, por tanto, los infocentros de manera aislada no pueden hacer frente a todas las demandas sociales que exige la ciudadanía. La transversalidad de la formación y servicios ofrece ayuda para que otras instituciones (bibliotecas, ONG, corporaciones locales) puedan colaborar en los mismos. Esto es particularmente relevante en lo que se refiere a la colaboración con las bibliotecas. No solo compartiendo la infraestructura o espacio, sino creando de manera conjunta un auténtico ecosistema de información en la que estos centros se conviertan en elementos de infomediación efectiva y asegurar el auténtico compromiso de su comunidad, factor que a tenor de Gould y Gomez (2010) determina el éxito de estas instituciones.

7. Para abordar la multidimensionalidad del concepto de empoderamiento digital, este estudio ha escogido tres colectivos clave en la inclusión social en Ecuador: mujeres, indígenas y personas con diversidad funcional. La red de infocentros facilita su estudio, puesto que cubre regiones menos favorecidas a nivel socioeconómico, donde estos colectivos necesitan de procesos que impulsen su empoderamiento personal y colectivo. Debido al escaso número de proyectos que han tenido como núcleo estos tres grupos meta, los autores recomiendan una revisión de los planes de formación de los infocentros que permita facilitar cursos específicos para estos colectivos.

8. Este trabajo puede ayudar a la toma de decisiones o a la mejora de los planes formativos de los infocentros como agente esencial en la lucha contra la brecha digital en Ecuador. El hecho de que la información provenga de la percepción de los propios gestores ayuda a la obtención de información más cercana que a través de simples datos estadísticos.

9. Los autores reclaman la necesidad de seguir reivindicando a través de la investigación estos espacios colaborativos comunitarios y de mejorar las sinergias con otras instituciones para el fomento del desarrollo local. Asimismo, estos han de alinearse con las perspectivas vitales actuales de los individuos, actualizándose y facilitando el espacio público para su empoderamiento y la toma de decisiones. 


\section{AGRADECIMIENTOS}

Esta investigación ha sido realizada gracias al apoyo y financiación de SENECYT (Secretaría Nacional de Educación Superior, Ciencia, Tecnología e Innovación de Ecuador).

\section{REFERENCIAS}

Alao, A.; Lwoga, T. E.; Chigona, W. (2017). Telecentres Use in Rural Communities and Women Empowerment: Case of Western Cape. En: Choudrie J., Islam M., Wahid F., Bass J., Priyatma J. (eds) Information and Communication Technologies for Development. ICT4D 2017. IFIP Advances in Information and Communication Technology, vol 504. Springer, Cham https://doi. org/10.1007/978-3-319-59111-7_11

Álvarez-Muñoz, P.; Hernández-Domínguez, C. (2016). Análisis longitudinal del Sistema Bibliotecario ecuatoriano. Revista Española de Documentación Científica, 39 (4): e155. https://doi.org/10.3989/ redc. 2016.4 .1327

Bailur, S. (2007). Using Stakeholder Theory to Analyze Telecenter Projects. Information Technologies and International Development, 3 (3), 61-80.

Benjamin, P.; Dahms, M. (1999). Socialise the modem of production - The role of telecentres in development. En: Gómez, R.; Hunt, P. (coords.), Telecentre evaluation: a global perspective. Quebec: International Development Research centre. Disponible en https://idl-bnc-idrc.dspacedirect.org/bitstream/handle/10625/15457/115668.pdf?sequence=1 [Fecha de consulta: 22/09/2018].

Breitenbach, M. C. (2013). Telecentres for sustainable rural development: Review and case study of a South African rural telecentre. Development Southern Africa, 30 (2), 262-278. https://doi.org/10.1080/037683 5X.2013.797229

Brito, S.; Socorro da Silva, R.; Mata, A.; Vijaykumar, E.; Rocha, C.; Abreu Monteiro, N.; Costa, J.; Francês, C. (2018). An approach to evaluate large-scale ICT training interventions. Information Systems Frontiers, 20(4), 883-899. https://doi.org/10.1007/s10796-0169705-3

CAF (Banco de Desarrollo de América Latina). (2013). Hacia la transformación digital de américa latina: las infraestructuras y los servicios tic en la región, 2013. Disponible en: http://scioteca.caf.com/handle/123456789/490 [Fecha de consulta: 19/09/2018]

Calderón, M. J.; Acuña, J. (2017). Conectividad rural y cambio social: los Infocentros Comunitarios en el Ecuador. Revista Publicando, 4 (11-1), 190-207. Disponible en: https://dialnet.unirioja.es/ejemplar/468070

Caridad Sebastián, M.; Martínez Cardama, S.; Puente Hernández, E. (2018). Bibliotecas universitarias en Ecuador: realidad y situación de los servicios de contenidos digitales. Investigación Bibliotecológica: archivonomía,

\section{ACKNOWLEDGEMENTS}

This research was financially supported by $\mathrm{Na}$ tional Secretary of Higher Education, Science, Technology and Innovation (SENESCYT).

bibliotecología e información, 32 (76), 111-129. https:// doi.org/10.22201/iibi.24488321xe.2018.76.57998

CEPAL. (2014). Los pueblos indígenas en América Latina. Avances en el último decenio y retos pendientes para la garantía de sus derechos. Disponible en: https:// repositorio.cepal.org/handle/11362/37050 [Fecha de consulta: 19/09/2018]

Colle, R.D; Roman, R. (1999). Communication Centres and Developing Nations: a state of the art report.

Comisión Europea. (2016). DigComp 2.0: The Digital Competence Framework. Retrieved from https:// ec.europa.eu/jrc/en/digcomp/digital-competence-framework [Fecha de consulta: 19/09/2018]

Fuente-Cobo, C. (2017). Públicos vulnerables y empoderamiento digital: el reto de una sociedad e-inclusiva. El profesional de la información, 26 (1), 5-12. https:// doi.org/10.3145/epi.2017.ene.01

Furuholt, B.; Sæbø, Ø. (2018). The role telecentres play in providing e-government services in rural areas: $A$ longitudinal study of Internet access and e-government services in Tanzania. The Electronic Journal of Information Systems in Developing Countries, 84(1), e12006. https://doi.org/10.1002/isd2.12006

García Álvarez, S. (2014). Sumak kawsay o buen vivir como alternativa al desarrollo en Ecuador. Aplicación y resultados en el gobierno de Rafael Correa (2007-2011). Universidad Complutense de Madrid. Disponible en: https:// eprints.ucm.es/24571/ [Fecha de consulta: 21/09/2018]

Gould, E. A. ; Gomez, R. (2010). Community Engagement \& Infomediaries: challenges facing libraries, telecentres and cybercafés in developing countries. iConference 2010, Feb 3-6, Urbana-Champaign, Illinois, USA.

Kirti; De Dipak. (2016). Increasing learning opportunities through digital empowerment. Journal of Global Communication, 9, 74-78.

Kumar, R.; Best, M. L. (2006). Social impact and diffusion of telecenter use: A study from the sustainable access in rural India project. The journal of community informatics, 2(3). Disponible en: http://ci-journal.org/ index.php/ciej/article/view/328/268 [Fecha de consulta: $21 / 09 / 2018$ ]

Lancaster, S. (1999). Telecentres and libraries: do they work together? Loughborough: Loughborough University, Department of Information Science.

Lwoga, E. T.; Chigona, W. (2018). Perception, usage and barriers towards the utilisation of the Telecentre among rural women in Tanzania. Journal of Information, Com- 
munication and Ethics in Society, 17 (1), 2-16. https:// doi.org/10.1108/JICES-01-2018-0004

Mäkinen, M. (2006). Digital empowerment as a process for enhancing citizens' participation. E-learning and Digital Media, 3(3), 381-395. https://doi.org/10.2304/ elea.2006.3.3.381

Martínez Cardama, S.; Gonsales Cordova, M.; Caridad Sebastián, M. (2015). La Sociedad del Conocimiento en el entorno europeo y latinoamericano: el caso de Ecuador y España. Ciência da Informação, 41, (2/3), 43-162.

Masiero, S. (2011). Financial vs. social sustainability of telecentres: mutual exclusion or mutual reinforcement? Electronic Journal of Information Systems in Developing Countries, 45 (1), 1-23. https://doi. org/10.1002/j.1681-4835.2011.tb00319.x
Ministerio de Telecomunicaciones y Sociedad de la Información. (2018). Libro Blanco de la Sociedad de la Información y el Conocimiento. Quito: Mintel.

Meshur, H.F.A. (2012). Telecenters as an information technology tool for development in urban and rural areas. International Journal of Arts \& Sciences, 5(1), 385-398.

Molina Benavides, L.S. (2015). Evaluación de las bibliotecas universitarias ecuatorianas: análisis del entorno y propuesta de un catálogo de indicadores. Universidad de Barcelona.

Morales García, A.; Caridad Sebastián, M.; García López, F. (2009). Los telecentros españoles: recursos, servicios y propuesta de indicadores para su evaluación. Information Research, 14(4), paper 420. Disponible en: http://www.informationr.net/ir/14-4/paper420.html [Fecha de consulta: 21/09/2018] 